\title{
Animal Health Surveillance Data Quality Assessment: The Case Study in Karsa Woreda, Jimma Zone, Oromia, Ethiopia, 2021
}

\author{
Chernet Balcha Jima (DVM) \\ Ministry of Agriculture Epidemiology Directorate, In-service Applied Veterinary Epidemiology Training \\ (ISAVET) Program, Oromia Regional State, Jimma Zone Livestock Resource Development Office
}

\begin{abstract}
Good surveillance data quality is vital for accurate planning and to apply timely and appropriate interventions. Data quality refers to completeness, accuracy and timeliness of data gathered. Despite consistent monthly reporting and clinical case registration, so far there is no document which could provide evidence for the quality status of surveillance data of the livestock sector at woreda level in Ethiopia. In action taken in Liberia after the quality audit, there was remarkable improvement in the quality of the data produced. Retrospective case study was conducted in Karsa woreda of Jimma zone Oromia regional state. The objective of the study is to assess the animal health surveillance data of the woreda and to provide new recommendations. The DOVAR format of the woreda from 2015-2020 and clinic case book were reviewed for the completeness, accuracy and timeliness. The records of data quality indicators in each variable of source document was counted and entered into Microsoft excel sheet. It then line listed and displayed in proportion. The overall data quality and related issues of the woreda were assessed by using a structured interview questions. The study shows that the assessed DOVARs are $83.3 \%$ complete. In the assessed reports there are $6.6 \%$ missing data, $35 \%$ inaccurate data and $31.6 \%$ late reports. This study also indicates that $89 \%$ of the sampled registered cases in the case book have missing data. The problem of accuracy in the case book is found to be $27.5 \%$. The surveillance data of the woreda have the problem of completeness, accuracy and timeliness. Data collectors didn't received training on surveillance. The woreda retain the collected data but do not analyze it. The woreda do not have clearly stated objectives for collecting surveillance data. These gaps lead them to have poor data quality DOVARs and case book. Therefore the woreda should develop clear objectives about the data that is needed; develop a clear plan about the best way of obtaining the data; use standardized formats that can capture the data required; train people on how to collect accurate and reliable data; store and retain data.
\end{abstract}

Keywords: Accuracy, Completeness, Data quality, Surveillance Data, Timeliness

DOI: $10.7176 / \mathrm{JMPB} / 72-01$

Publication date: January $31^{\text {st }} 2022$

\section{BACKGROUND}

Good surveillance data quality is vital for accurate planning and to apply timely and appropriate interventions. Data is a collection of items of information. It can be defined as the elements of measurements recorded during data collection. It is the collected data that create information when it is further processed which will then improve the knowledge of end users. Knowledge is then assessed to improve the understanding of the researchers which provides wisdom. Wisdom is required to take evidence based decisions for action (FAO).

Data quality refers to completeness, accuracy and timeliness of data gathered and that they convey the intended meaning. There are data quality principles which are essential to ensure data quality. It begins by ensuring that data is gathered in a standard way. To ensure good quality data it is important to have clear objectives about the data that is needed; develop a clear plan about the best way of obtaining the data; use standardized formats that can capture the data required; train people on how to collect accurate and reliable data; store and retain data so that it can be managed for future use and provide feedback to stakeholders using the data (FAO). ISAVET program of FAO is designed to link the theoretical data quality audit principles learnt in the classroom with the actual work place animal health surveillance data.

The study was conducted in Karsa woreda of Jimma zone Oromia regional state, Ethiopia. The woreda is located $325 \mathrm{~km}$ from Addis Ababa in the southwestern direction. According to Jimma zone livestock development office there are thirty two kebeles (thirty PAs and two kebeles) in the woreda. Most recent record indicates that the total population of the woreda is 221,945 (107,725 males and 114,220 females).According to the data recorded in the woreda office the livestock population of the woreda in 2013 E.C. is; cattle: 325,025 ; horse: 8,509 ; donkey: 18,550 ; mule: 2,075 ; sheep: 75,851 ; goat: 39,316 ; poultry: 85,492 and dog: 4,477 . There is $90 \%$ woynadega and $10 \%$ dega agro ecology with temperature range of $15-27^{\circ} \mathrm{c}$. The woreda has one "B" type; one " $\mathrm{C}$ " type; four " $\mathrm{D}$ " type and thirteen non standard veterinary clinics/posts. There is one private veterinary pharmacy in the woreda. Veterinary workforce of the woreda is described in the table below: 
Table 1: Veterinary workforce and Infrastructure of Kersa woreda

\begin{tabular}{|c|c|c|c|c|c|c|c|}
\hline Qualification & $\overline{\mathrm{MSc}}$ & $\overline{D V M}$ & $\overline{B V S c}$ & AHA & VLT & AHT & Total \\
\hline Number & 1 & 3 & 4 & 23 & 1 & 1 & 33 \\
\hline \multirow{2}{*}{ Vet posts/ clinics } & Stan & & & & \multirow{2}{*}{ Non standard } & & \\
\hline & $\mathrm{A}$ & $\mathrm{B}$ & $\mathrm{C}$ & $\mathrm{D}$ & & & \\
\hline Number & - & 1 & 1 & 4 & 13 & & 19 \\
\hline
\end{tabular}

The data quality audit in a case study conducted in the public health service of Grand Bassa County, Liberia helped to identify key factors influencing surveillance data quality in the district. In action taken after the quality audit, there was remarkable improvement in the accuracy and completeness of the data produced (Joseph A., et. al., 2015). Despite consistent monthly reporting and clinical case registration, so far there is no document which could provide evidence for the quality status of surveillance data of the livestock sector at woreda level in Ethiopia. This study was conducted to assess the animal health surveillance data quality of the woreda and to provide new significant recommendations which will help to apply basic principles of ensuring data quality. This will finally let to improve the previous practices and produce quality data which will be an input for other stakeholders.

\subsection{General objective}

The general objective of the study was to assess and describe the surveillance data quality and related gaps in Kersa woreda Jimma zone Oromia regional state Ethiopia and provide recommendations.

\subsection{Specific objectives}

The specific objectives of the study were to:

$>$ Assess how data is collected and managed in the woreda;

$>$ Assess the surveillance data and information flow;

$>$ Review archived DOVAR reports and case books to assess the quality of data;

$>$ Assess the awareness of the stakeholders about data use and

$>$ Assess the link of laboratory with field investigation.

\section{METHODS}

\subsection{Study Area}

The study was conducted in Karsa woreda of Jimma zone Oromia regional state, Ethiopia. The woreda is located $325 \mathrm{~km}$ from Addis Ababa in the southwestern direction. There are thirty two kebeles (thirty PAs and two kebeles) in the woreda. Most recent record indicates that the total population of the woreda is $221,945(107,725$ males and 114,220 females). Serbo type "B" veterinary clinic is selected for the study.

\subsection{Data collection and processing}

Retrospective study with quantitative and the qualitative approaches is used to conduct the study. Key informant interview, retained DOVAR report and clinic case book data quality assessments were applied. A checklist was developed and used to interview the responsible woreda professionals who are involved in animal health surveillance. The responses obtained were evaluated according to the data quality principles. Archived disease outbreak and vaccination activity reports (DOVAR) from 2015-2020 were reviewed for the quality assessment based on data quality principles and national standards. Clinic case book were also reviewed for the completeness and accuracy. The data quality indicators and major findings were counted and entered into Microsoft excel sheet.

\subsection{Data analysis}

The recorded data were analyzed using Microsoft excel and presented using graphs. The responses for the queries obtained from the interviewee were evaluated according to the data quality principles.

\subsection{Study Variables}

Completeness, accuracy and timeliness are the attributes considered in this study.

Completeness: the percentage of blank or unknown data, not zero/missing. All data should be in a disaggregated form to permit further analysis.

Accuracy: the percentage of data variables on the collection form without an error: examples - missing data, incorrect coding, transposed error, incorrect units, incorrect/ inconsistent format.

Timeliness: the percentage of reports from the sub locals, and facilities that were received on time. 


\section{RESULTS}

\subsection{Information obtained from the interview}

\subsubsection{Surveillance data source, collection, flow and analysis}

Kebele animal health workers collect surveillance data from the farmers. They use a standard reporting format for vaccination and other animal health service activity report. However, the occurrence of disease outbreak in a peasant association is reported to the woreda by phone and/or orally. The woreda then use DOVAR format to submit to regional veterinary laboratory and zone livestock office. The responsible professional in the woreda assess the completeness, accuracy and timeliness of the reports and take corrective measures.

Surveillance data flow of the woreda can be described in the following diagram:

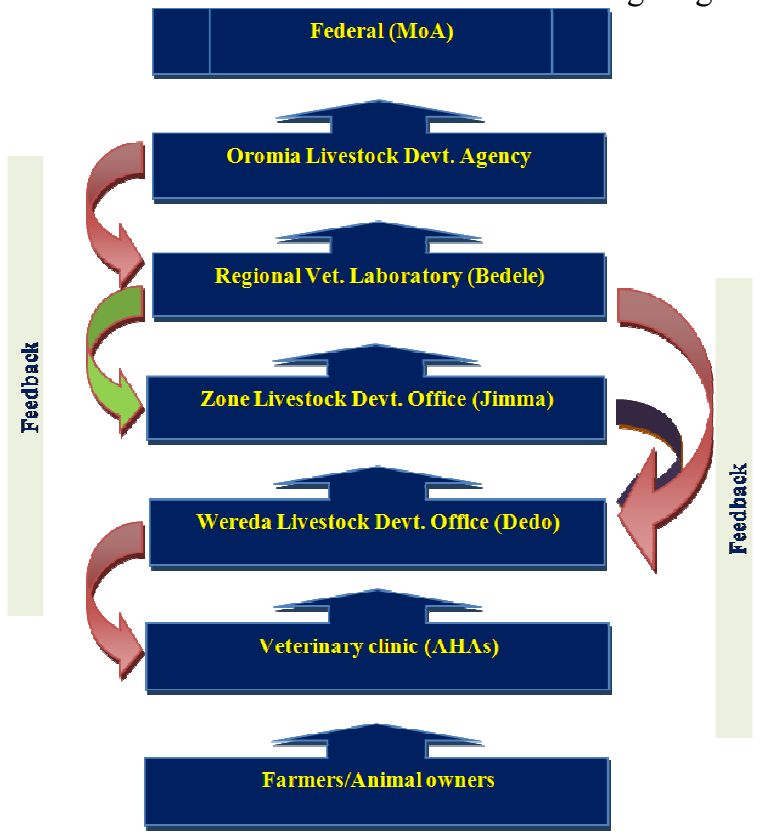

Figure 8: Animal health surveillance data flow in Kersa woreda

This study consider DOVAR and clinic case registration book as surveillance data. The woreda retain these data in their office as permanent document. However the woreda did not analyze the data they stored in animalplace-time.

\subsubsection{Surveillance data benefit (usefulness)}

Animal health workers at the woreda level did not received training about surveillance. This gap leads these professionals not to understand the benefit of surveillance data.

\subsubsection{Awareness of data use by stakeholders}

Though the interviewee said that the surveillance data (case book) is used to know about the disease distribution, to refer back previous cases for different purposes, to build trust of owner; the woreda have no clearly stated objectives for collecting surveillance data. The clinic case recorder said that the case book data seem to have importance but not sure. According to the responsible woreda professional's response surveillance data collectors have no awareness on what will be done with the collected data.

\subsubsection{Use of laboratory in field investigation/surveillance}

Bedelle veterinary regional laboratory is the responsible institution to provide diagnostic support for the woreda. However, no sample was submitted to the laboratory during the past six year period. Thus it is not possible to speak about the time required to collect and deliver samples to the laboratory and the time required to receive feedback about laboratory test result. This shows that there is weak link between laboratory and field investigation.

\subsection{Assessment of DOVARs}

From the total 72 DOVAR format which are expected to be sent every month in the past six years, only 60 reports were found in the woreda office. Reports sent in 2015, 2016, and 2017 were complete while 11, 9 and 4 reports were encountered for 2018, 2019 and 2020 respectively. The available reports can be described in the following (Table: 2 ). 
Table 2: Available DOVAR formats between 2015 and 2020 in Kersa woreda.

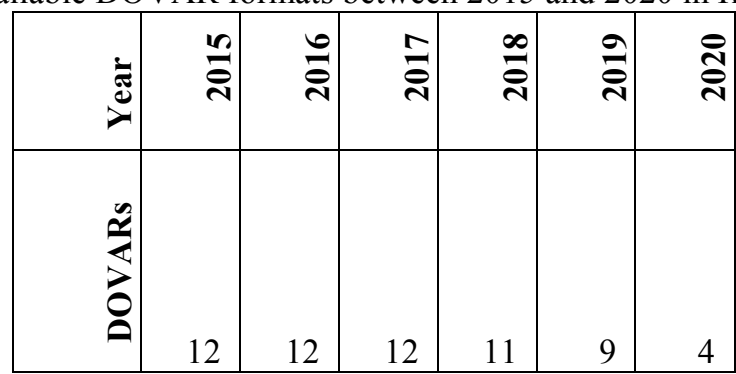

These available reports were assessed against the key data quality indicators. There were only $6.7 \%(4 / 60)$ (two LSD and two FMD) outbreak reports in the past six years. The rest of the reports $(93 \%)$ are zero reports. It is reported through the usual informal way to the woreda and the woreda veterinarians conduct field investigation. Out of sixty (60) reviewed reports $6.6 \%(4 / 60)$ have missing data while $35 \%(21 / 60)$ have a problem of accuracy. On the other hand 31.6\% (19/60) of reports have a problem of timeliness.

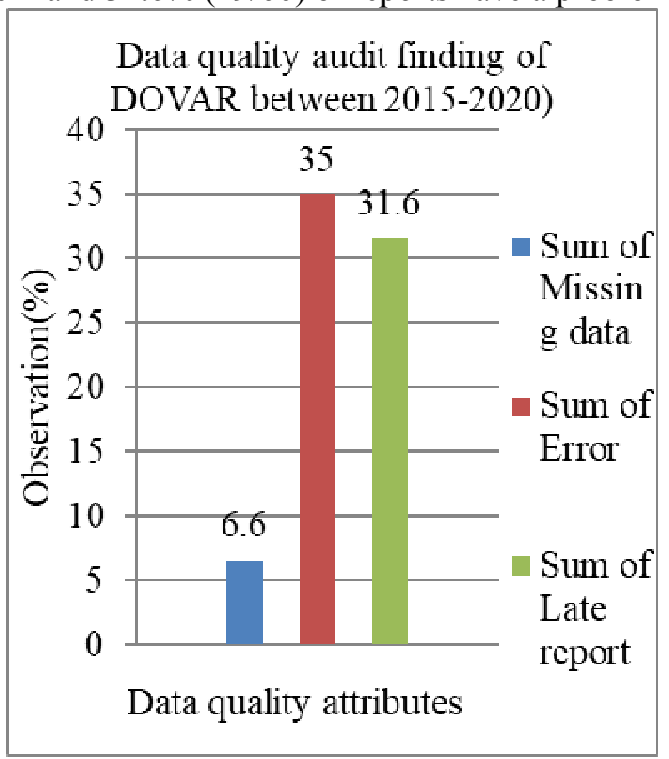

Figure 1: Data quality audit findings from DOVAR of Kersa woreda

From the total missing data counted, $25 \%(1 / 4), 50 \%(2 / 4)$ and $25 \%(1 / 4)$ is related to the name of reporting woreda, phone number of the reporting person and reporting date respectively.

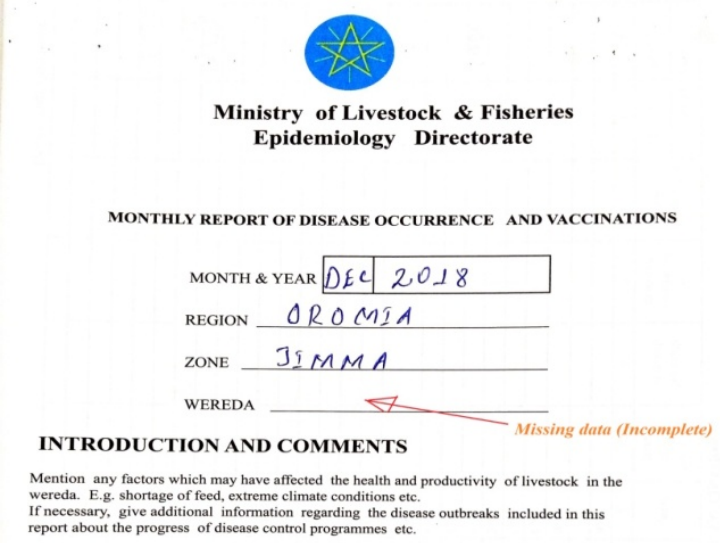

Figure 2: Evidence of missing data (the reporting woreda name) 

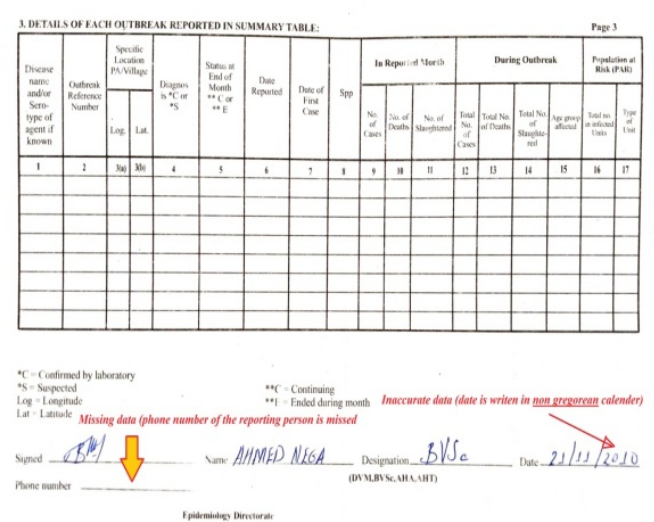

Figure 3: Evidence of missing data (the phone no. of the reporting person) and Date in Non Gregorian calendar

From the total $35 \%$ accuracy problem found $42.8 \%(9 / 21)$ of the reports have typing error (writing the general information on page 1 in small letter which was expected to be written in capital letter). On the other hand $28.5 \%(6 / 21)$ of the reports have no official stamp on it and $14.2 \%(3 / 21)$ have ineligible writing. As regards to the timeliness $31.6 \%$ (19/60 of reports were received lately.

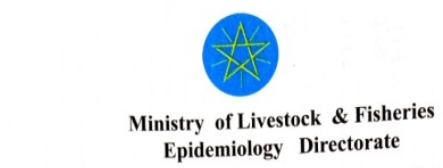

MONTHLY REPORT OF DISEASE OCCURRENCE AND VACCINATIONS

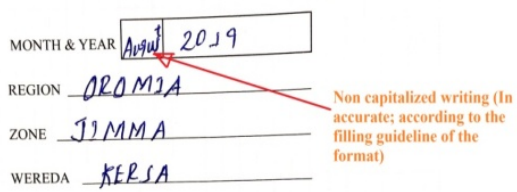

INTRODUCTION AND COMMENTS
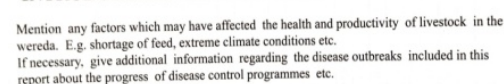

Figure 4: The month is written in non capitalized letter

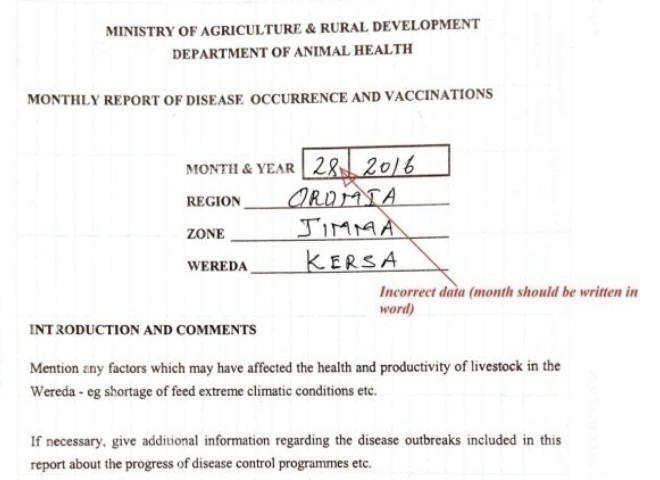

Figure 5: Date is written in the blank space of month

\subsection{Assessment of clinic case registration book}

From the total of 200 reviewed cases in the case registration book of Serbo type "B" veterinary clinic of the woreda $89 \%(178 / 200)$ were found to have missing data and $27.5 \%(55 / 200)$ were found to have errors (Figure 2). 


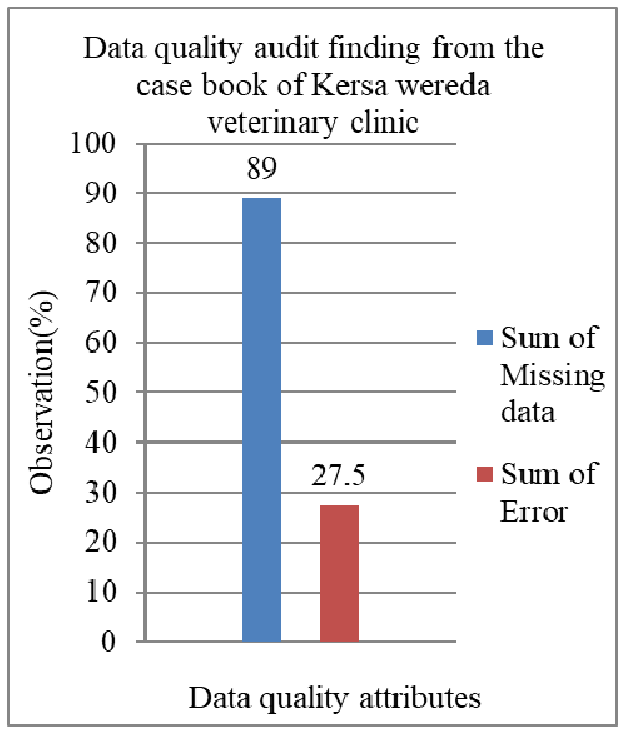

Figure 6: Data quality audit findings from case registration book of Serbo clinic, Kersa woreda

In the case book some of the observations have more than single missing data. Out of the total missing data found $83.1 \%$ (148/178), 35.3\% (63/178), 28.08\% (50/178), $11.7 \%$ (21/178), 9.5\% (17/178), 2.8\% (5/178), $1.6 \%$ $(3 / 178)$ and $1.12 \%(2 / 178)$ are related to the age, clinical finding, diagnosis, treatment, breed, history, sex and species respectively. The highest missing data is observed under the column of age (83.1\%). The lowest missing data is counted from the column of species and sex.

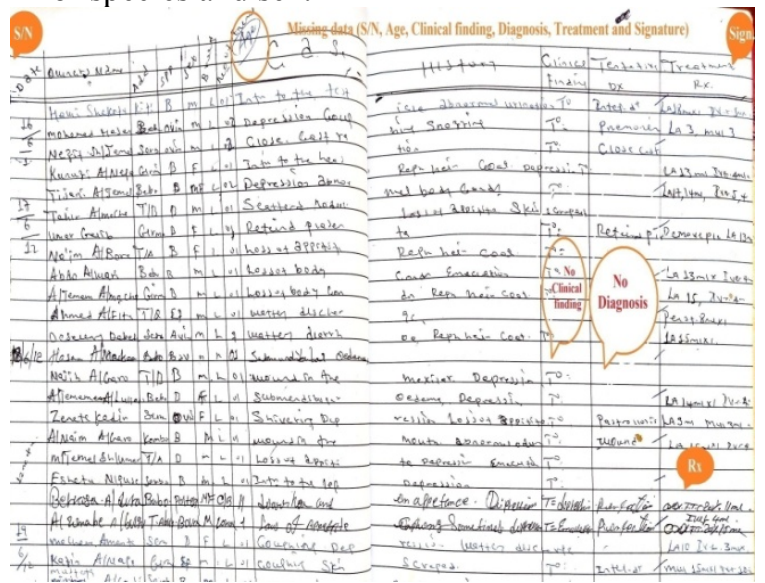

Figure 7: Missing data and errors found in the case book of Kersa woreda Serbo veterinary clinic

On the other side out of the errors found in the case recording book $74.5 \%(41 / 55), 18.1 \%(10 / 55)$ and $3.6 \%$ $(2 / 55)$ were observed under the column of treatment, age and sex respectively. Similar to the category of sex $3.6 \%(2 / 55)$ error is detected from the column of diagnosis.

\subsection{Information obtained from other observations during the study period}

The reviewed disease outbreak and vaccination activity reports (DOVARs) were not stored in an easily accessible way in the office. There is poor file storage mechanism in the woreda.

\section{DISCUSSION}

The quality of data is dependent on the underlying data collection, management and reporting systems; stronger systems should produce better quality data. This study revealed that there is a defect on the quality of animal health surveillance data of Kersa woreda. There is no well documented study relating with surveillance data quality audit yet which may help us to compare with and discuss the results of the present study. Nevertheless, most institutions might need some improvements in this area. More than $93 \%$ of the assessed reports were zero reports.

There is missing data, inaccurate data and problem of timeliness in the assessed DOVARs of Kersa woreda livestock development office. Majority of the data quality problems found were restricted to page 1 (general information) and page 3 (the details of the outbreak reporting person) of the format. The highest $(42.8 \%)$ inaccurate data is non capitalized writing of the general information on page 1 of DOVAR format. According to the filling guidelines of the format, the general information should be capitalized. Reports should be received 
before $18^{\text {th }}$ day of every month. From the total reports assessed during the study period $31.6 \%$ is reported after the deadline. This late reporting is probably because of lack of preparedness; which may be related with the availability of the reporting format in the office and delay of reports from the kebeles. Inaccurate data that entered in the format (35\%) indicates that there is a gap in understanding the filling guideline.

This study also indicates that $89 \%$ of the sampled registered cases in the clinic case registration book have missing data. According to Livestock Development Agency, the standard veterinary clinic case book format include serial number, date, owner's name, PA, species, breed, age, sex, history, clinical signs, diagnosis, treatment and signature. But some of their case registration book pages lack columns for $\mathrm{S} / \mathrm{N}$, age, breed and signature. The highest missed data is age $(83.1 \%)$ which is followed by clinical finding $(35.3 \%)$. Most of the pages in the case book do not have a column for "age". There are also some pages lacking a column of "clinical finding". Diagnosis (28.08\%) is the other variable missed. After filling the whole variables in the case book, the case book recorder miss the clinical finding and diagnosis part because of different reasons. Some of the reasons are negligence, thinking that it is time consuming, having limitation to determine the diagnosis only by depending on inadequate diagnostic technique. History, sex and species are the least missed data in the case book.

The problem of accuracy or error is found to be $27.5 \%$ of the reviewed observations in the case book. Out of this the highest $(74.3 \%)$ is related with treatment. Mostly the column under treatment is left blank or recorded with incomplete information. Though the clinician try to justify that it is because of small space in the case book, this problem emerges from lack of awareness on what will be done with the collected data. In fact the space in the case book is not enough to write the whole information in a single row of the individual case. However this problem can be solved by preparing printed case paper for each case. Negligence is may be the other reason for these problems. The lowest inaccurate data $(3.6 \%)$ is found in association with diagnosis. Spelling errors were observed while writing the name of the disease diagnosed. There is also mismatch of the history, diagnosis and treatment.

The surveillance data of the woreda is collected from the farmer at clinic level and shared to the woreda livestock development office, zone livestock development office, Bedelle regional veterinary laboratory, Oromia livestock development agency and ministry of agriculture. The clinician interviewed said that they did not received training about animal health surveillance. They do their job with their own knowledge and experience. At the data collection source there is no standard data collection format for an outbreak. Animal health workers at clinic level report an outbreak orally. This leads to lack of documented outbreak reports at the clinic level which can be utilized by different stakeholders. Therefore, the woreda should develop disease outbreak reporting format that can fit with DOVAR. However, there is standard reporting format for their monthly activity report. But still their case book variables do not exactly match with the standard veterinary clinic case book variables (OLFDO, 2017). Though the coordinator reply that they do identify missing values and errors in the data for each variable; there is still missing data and error in the DOVARs and the case book.

The woreda should analyze the data they collect in animal-time-place. Analyzing the collected surveillance data is important to take necessary actions depending on the findings. The woreda has its own objectives and goals with regard to providing good veterinary service. However, they should also develop clearly stated objectives why they are collecting animal health surveillance data. They should effectively compile reports and store in their office as permanent document. In this way they can improve their data management. The data collectors also have inadequate awareness on what will be done with the collected data.

Bedelle veterinary regional laboratory is the responsible laboratory to provide diagnostic support for Kersa woreda. However no sample was submitted to this laboratory during the past six years. This shows that there was no chance of combining laboratory and field data for analysis. Nevertheless, the indicated laboratory has been undergoing supervision to evaluate and provide recommendations about the laboratory work of the woreda clinic.

Animal health team coordinators of the woreda were unable to provide the required DOVAR reports quickly. This is because DOVARs were not stored in an easily accessible way in the office. This indicates that the file storage mechanism of the woreda is weak.

\section{CONCLUSION}

A strong surveillance system needs to have an effective way of collecting data, analyzing, and interpreting quality surveillance data so that to act accordingly for the welfare and health of the animal and public health as well. To ensure early detection and response to diseases of concern as well as to maintain the health of the livestock there should a strong surveillance at the lowest level. The flow of Kersa woreda animal health surveillance data begins from the farmers. The clinicians do not have outbreak reporting format. Since the data collectors have inadequate awareness on data usefulness, they thought that careful data collection is less important. The surveillance data of the woreda have the problem of completeness, accuracy and timeliness. The woreda make an effort to identify missing data, errors and the timeliness of the reports. However it is not a formally organized activity. They retain the collected data in their office but do not analyze it in animal-placetime. The woreda does not have clearly stated objectives for collecting surveillance data beyond listing some 
functions of the collected data. The link of laboratory and field investigation is week. The data collectors didn't received training on surveillance. This lack of training leads the woreda to have poor data quality case book and DOVARs.

\section{RECOMMENDATION}

Depending on the above conclusion, I recommend Kersa woreda livestock development office animal health team that they should:

* Have clearly stated objectives for collecting animal health surveillance data.

* Develop a clear plan for obtaining surveillance data.

* Regularly analyze data at woreda and share information for stakeholder so that to take action based on the findings.

* Provide training for the frontline animal health workers on surveillance so that to get complete, accurate and timely report.

* Improve the link of laboratory to support investigation of outbreaks and capacitate woreda clinics to conduct basic laboratory tests.

* Develop a standard format for kebeles for the reporting of outbreak data that can be aligned with the DOVAR format.

* Zones and regions (Agency \& Laboratory) should provide regular feedback and share summary reports on DOVAR.

* Develop well designed case book or individual case paper for suitable case registration in the clinics

* Surveillance data quality assessment should be conducted in a periodic manner.

\section{REFERENCE}

1. Food and Agriculture Organization of the United Nations: Frontline In-Service Applied Veterinary Epidemiology Training Curriculum Manual Participant Guide. Texas A \& M Agri Life - Institute for Infectious Animal Diseases

2. Hong Ch., David H., Ning W. and Ping Y. (2014): A Review of Data Quality Assessment Methods for Public Health Information Systems: International Journal of Environmental Research and Public Health, 11, ISSN $1660-4601$

3. Jimma zone livestock development office: Fundamental documents of the entire woreda in 2020.

4. Joseph A. F., Maame P. A., Peter A. A., Casey D. H., Meeyoung M. P., Thomas K. N. (2015): Conducting a Surveillance Data Quality Audit in Grand Bassa County, Liberia, November 2015, Liberia Field Epidemiology Training Program, Monrovia, Liberia; 2Rollins School of Public Health, Emory University, Atlanta, USA; 3Ministry of Health, Monrovia, Liberia.

5. Oromia Livestock and Fishery Development Office: Veterinary Service Delivery Guideline for Veterinary Clinics of Oromia April 2017.

6. The Global Fund: Data quality audit tool, Guidelines for implementation.

\section{ANNEXES}

\section{Annex: 1 \\ Interview Question \\ Ministry Of Agriculture Epidemiology Directorate \\ In-service Applied Veterinary Epidemiology Training (ISAVET) Program}

Dear respondent!

I am currently conducting a study entitled as "Assessment of Animal Health Surveillance Data Quality in Karsa District, Jimma, Ethiopia". The main objective of this interview is to assess the animal health surveillance data quality of Kersa district. I kindly request you to give genuine responses.

\section{Description of surveillance system of the woreda}

1.1 Describe the surveillance data flow of the woreda using diagram(farmer to vet clinic/post to woreda, and to zones and other stakeholders)

\section{Data collection}

2.1 Who collects the surveillance data?

\subsection{What are the sources of data?}

2.3 Did data collectors receive training on surveillance in the last 3years? Yes 
2.4 Is standard reporting format used at the data collection source? Yes No

2.5 How is the data submitted from the source to the woreda?

2.6 Do you identify missing values (assess completeness) in the data for each variable? Yes No

2.7 Do you identify errors (assess accuracy/correctness) in the data for each variable? Yes No

2.8 Do you identify timely, late or absent reporting from the reporting sites/clinics/kelas? Yes No

2.9 Describe any actions that have been taken by the woreda to correct late, absent, or incomplete reporting from the reporting sites/clinics/kelas.

\subsection{Describe surveillance data storage mechanisms by the woreda.}

\section{Data analysis}

3.1 Do you analyze the data you collect in animal-place-time?
Yes
No
3.2 If yes, which display methods do you use (table, map, graphs, or charts)?

\section{Surveillance data benefit (Usefulness)}

a. Describe the frequency and kind of reports used to share data from one level to the next (the farm or village to vet clinic/woreda; the vet clinic/post to woreda; and woreda to zone).

b. How many surveillance data reports led to conducting field investigations?

c. Describe the feedback mechanism by the woreda on surveillance data quality and performance to vet clinics/posts and farmers.

\section{Awareness of data use by stakeholders}

5.1. Does the woreda have clear objectives for collecting the surveillance data?

Yes
5.2. Do the data collectors have awareness on what will be done with the collected data?
Yes

Yes
6. Use of Laboratory in field investigation/surveillance

6.1. Which veterinary laboratory does provide diagnostic support for you?

6.2. How many samples were submitted to the laboratory during the past 3 years?

6.3. What is the minimum and maximum time (days, hours) required to collect and deliver samples to the laboratory during the past 3 years?

6.4. What is the minimum and maximum time (days, hours) required to receive feedback about laboratory test results past 3 years?

6.5 Describe if and how laboratory and field data are combined for analysis? 
Annex: 2

Standard disease outbreak and vaccination activity report (DOVAR) format (Page 1)

\section{Ministry of Agriculture}

Epidemiology Directorate

MONTHIY REPORT OF DISEASE OCCURRENCE AND VACCINATIONS

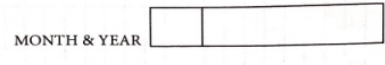

REGION

ZONE

WEREDA

INTRODUCTION AND COMMENTS

Mention any factors which may have affected the health and productivity of livestock in the

If necessary, give additional information regarding the disease outbreaks included in this
report about the progress of

Ensure Timely and Regular Reporting for Better Disease Control!

Epidemiology Directorate

(Page 2 of DOVAR Format) 


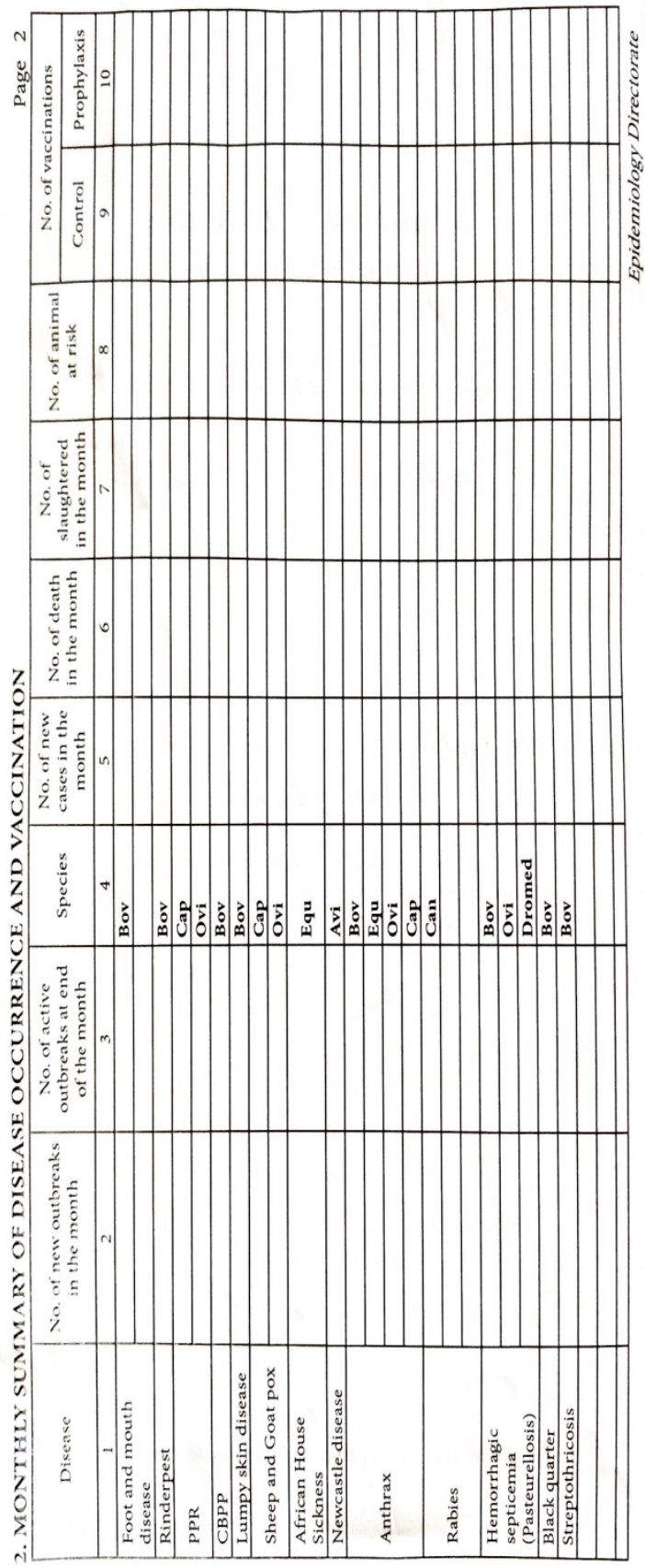

(Page 3 of DOVAR Format) 


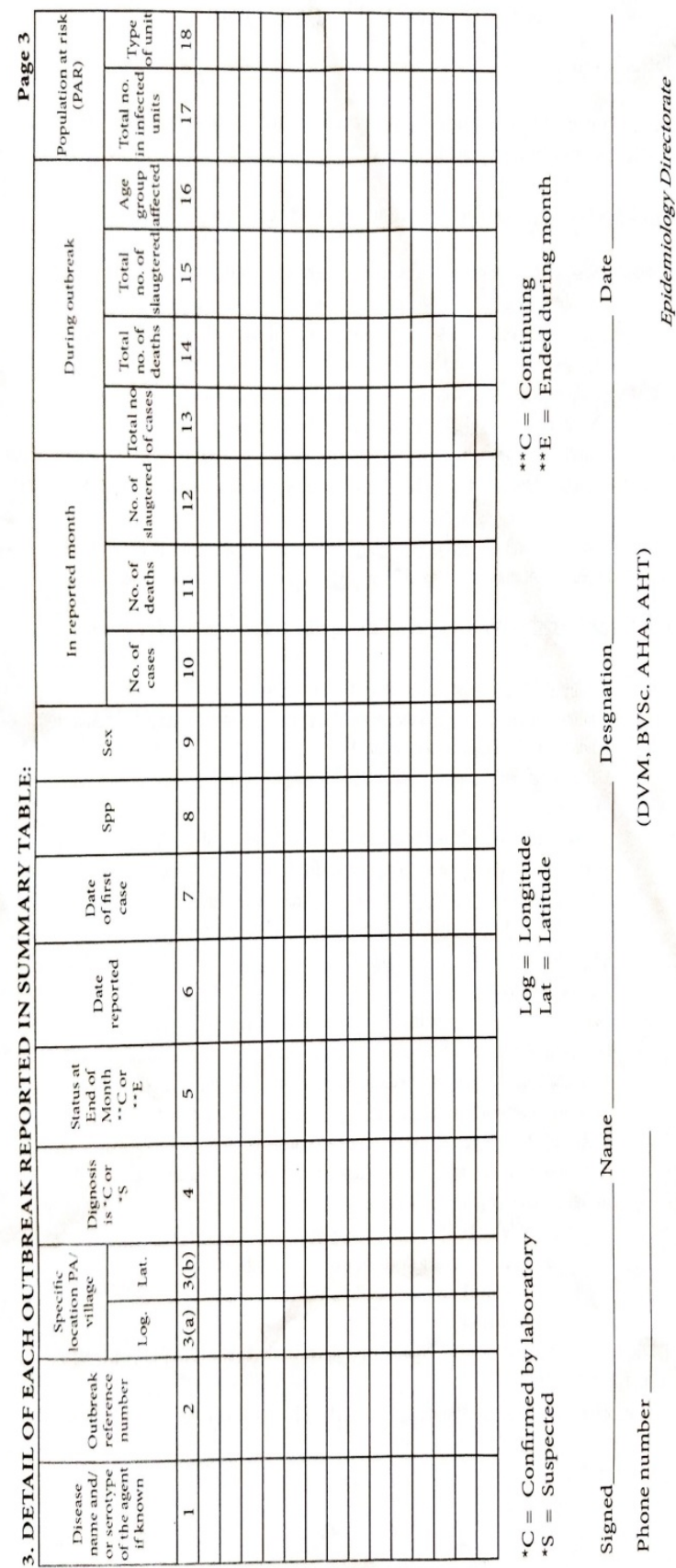

Page 4 of DOVAR Format 


\section{Page 1. General}

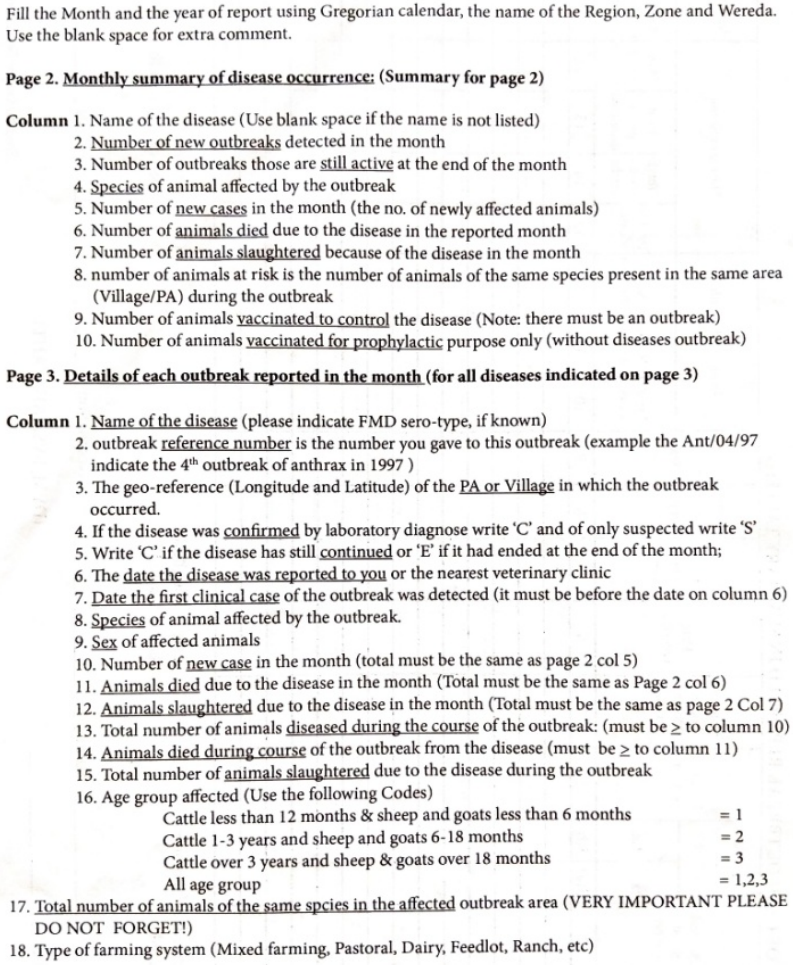

Total number of animals of the same spcies in the affected outbreak area (VERY IMPORTANT PLEASE DO NOT FORGET!)

18. Type of farming system (Mixed farming, Pastoral, Dairy, Feedlot, Ranch, etc)

Annex: 3

Standard case registration book format for veterinary clinics of Oromia (From page 12 of the guideline)

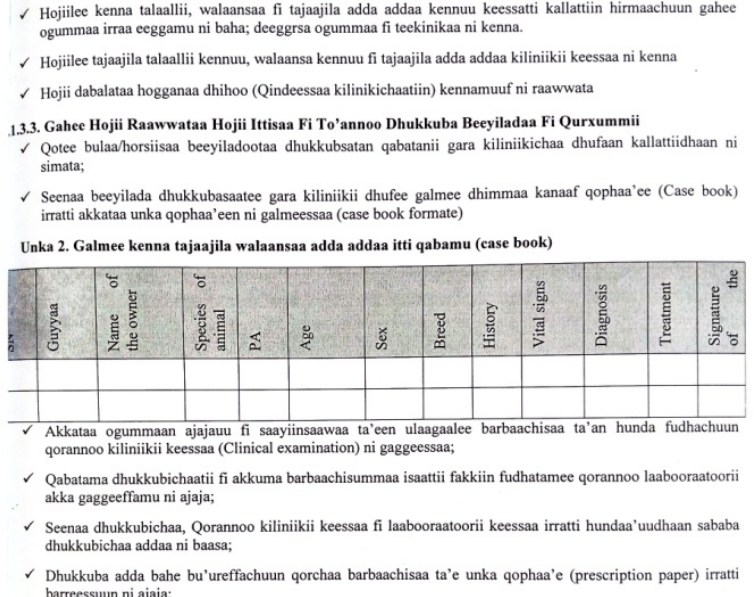

